Article

\title{
Long-Term Satisfaction and Patient-Centered Outcomes of Deep Brain Stimulation in Parkinson's Disease
}

\author{
Jessica A. Karl ${ }^{1, *}$, Bichun Ouyang ${ }^{1}$, Kalea Colletta ${ }^{2}$ and Leo Verhagen Metman ${ }^{1}$ \\ 1 Movement Disorder Section of Neurological Sciences, Rush University Medical Center, \\ 1725 W. Harrison Street, Suite 755; Chicago, IL 60612, USA; bichun_ouyang@rush.edu (B.O.); \\ lverhage@rush.edu (L.V.M.) \\ 2 Department of Neurological Sciences, Loyola University Medical Center, 2160 S. 1st Ave.; Maywood, \\ IL 60153, USA; kcolle2@gmail.com \\ * Correspondence: jessica_karl@rush.edu; Tel.: +01-312-563-2900
}

Received: 21 March 2018; Accepted: 30 March 2018; Published: 1 April 2018

\begin{abstract}
Bilateral subthalamic nucleus (STN) deep brain stimulation (DBS) is an effective and proven treatment option for patients with advanced Parkinson's disease (PD). Long-term outcomes ( $>5$ years) have demonstrated sustained improvement in objective motor symptoms; however, few studies have evaluated patient-centered outcomes other than quality of life (QOL). A locally developed DBS-patient-centered outcomes questionnaire was administered to PD patients $>5$ years post-DBS. All questions were scored on a ten-point scale, whereby 0 represented the most 'positive' answer and 10 the most 'negative' answer. Pre-operative scales were repeated at the time of survey. Fifty-two patients (mean $8.2 \pm 2.6$ years post-DBS) were included. Satisfaction was high with median score (range) of 1/10 (0-8) at the time of survey. Patients endorsed having made the correct decision by undergoing DBS, with a score of $0(0-10)$, would choose to have DBS again, with a score of $0(0-10)$, and would recommend DBS to others, with a score of $0(0-10)$. Pre-operative expectation target was set at a high level with a score of 2 (0-10). Parkinson's Disease QOL (PDQ-39) Questionnaire Summary Index (SI) scores were, mean (SD), $2.1(18.2)$ above baseline $(p=0.44)$. Those with worsening in PDQ-39-SI scores had less satisfaction with DBS $\left(r_{s}=0.57, p \leq 0.0001\right)$. This is the first study to assess long-term patient satisfaction with STN DBS. We are currently collecting data prospectively to confirm the results of these preliminary findings.
\end{abstract}

Keywords: deep brain stimulation; Parkinson's disease; subthalamic nucleus; patient-centered outcomes; quality of life; satisfaction; long term

\section{Introduction}

Bilateral subthalamic nucleus (STN) deep brain stimulation (DBS) is an effective and proven treatment option for patients with advanced Parkinson's disease (PD). Long-term outcomes ( $>5$ years) have demonstrated sustained improvement in objective motor symptoms [1-3]. There have been few studies evaluating patient-centered outcomes other than quality of life (QOL). Health-related QOL appears to be variably maintained and can differ between individual domains [4,5]. Hasegawa et al. [6] did evaluate patients' perspectives of DBS outcomes in the short term (1 year) and found no correlation between Parkinson's Disease QOL (PDQ-39) Questionnaire scores and overall satisfaction, but did find that preoperative counseling of patients' expectations did positively affect patient satisfaction. Unrealistic expectations of DBS surgery can lead to reduced satisfaction amongst patients and caregivers, even in the face of 'objective' motor improvement. There has been no exploration of satisfaction and expectations in the long term ( $>5$ years) and overall long-term patient-centered outcomes of DBS are lacking. The Patient- 
Centered Outcomes Research Institute (PCORI) stipulates a goal of, " ... evaluating questions and outcomes meaningful and important to patients and caregivers [7]." Accordingly, in this study we determined long-term patient satisfaction, QOL, and self-reported disability after STN DBS. In addition, predictors of satisfaction and the relationship between satisfaction and changes in QOL and symptom severity were investigated.

\section{Methods}

PD patients who received bilateral STN DBS at Rush University Medical Center (RUMC) a minimum of five years earlier were identified. The study was approved by the Rush University Institutional Review Board (ID: 14082201; Date of approval 15 October 2014). Each participant provided informed consent. At the time of surgery, extensive multi-disciplinary testing had been completed by the RUMC DBS team to determine patients' eligibility for DBS surgery, including evaluations by a movement disorder neurologist, neuropsychologist and neurosurgeon. Standard rating scales, including Unified Parkinson's Disease Rating Scale (UPDRS) I-IV, Schwab and England scale (S\&E), PDQ-39, as well as pre-operative demographics and Levodopa equivalent daily dose (LEDD) were available for review in all cases. After study entry, current demographics were obtained and the PDQ-39, UPDRS-I, UPDRS-II, S\&E and UPDRS-IV were repeated to evaluate changes in QOL, mood and cognition, disability, and complications of therapy. In addition, current LEDD was calculated.

\section{Patient-Centered Outcomes}

To evaluate post-operative satisfaction, a locally developed DBS patient-centered outcomes questionnaire (RUSH-DBS-Q) was administered (Table S1). The RUSH-DBS-Q is divided into two sections. The first section focuses on patients' pre-operative expectations, their theoretical decision to undergo DBS again, their sense of timing of DBS, their confidence in recommending DBS to others, and their overall satisfaction with DBS. The second section asks patients to rate the severity of their motor (dyskinesia, motor fluctuations, tremor, rigidity, bradykinesia, gait, balance, dystonia) and non-motor (cognitive impairment, depression, apathy, insomnia, excessive daytime sleepiness, pain, PD medication side effects) symptoms before surgery and at the current time. All questions are scored on a ten-point scale, whereby 0 represents the most 'positive' answer and 10 the most 'negative' answer. Patients and their caregivers filled out the RUSH-DBS-Q an average of eight years post-DBS surgery, so recall and other bias is inherent. However, a substantial portion of the questionnaire targeting post-operative outcomes was completed at the appropriate time.

The questionnaires were mailed to patients prior to the interview to give patients and caregivers the chance to familiarize themselves with the content, and once they indicated to be prepared the interview was conducted. Data were collected in the outpatient clinic or over the phone according to patient/caregiver preference. Patients and caregivers worked together to answer all questions.

\section{Statistical Analysis}

Descriptive analysis (mean, median, standard deviation, range) was obtained for each variable. To evaluate predictors of outcome, the pre-operative UPDRS-III sub scores were grouped into the following factors: axial (items 29-30), resting tremor (item 20), postural tremor (item 21), bradykinesia, (items 23-26) and rigidity (item 22) [8]. Comparison of clinical data pre-operatively and at time of study was completed using the paired t-test for normally distributed data and the Wilcoxon signed rank test for non-parametric data (statistical significance determined at $p<0.05$ ).

Patient ratings on the RUSH-DBS-Q of motor and non-motor symptoms before surgery and at the current time were compared. If scores were identical these symptoms were labeled "no change", if scores were lower at the current time the symptoms were labeled "better" and if scores were higher those symptoms were labeled "worse".

Spearman correlation analysis was completed to evaluate the relationship between post-operative satisfaction scores and reported symptom severity, pre-operative expectations, and pre-operative 
response to levodopa (change in baseline UPDRS-III-OFF to baseline UPDRS-III-ON). The relationship between change in LEDD from pre-DBS to current time and apathy was also investigated.

\section{Results}

\subsection{Participants}

Ninety-four PD patients were identified who received simultaneous bilateral STN DBS a minimum of five years earlier. Fifty-two patients (thirty-one male, twenty-one female) were enrolled, thirty-one were lost to follow up of whom at least twelve were deceased, six were non-English-speaking, five refused to participate, and one had been explanted. Patients who received unilateral STN or DBS in other targets were excluded from participation.

\subsection{Rating Scales and LEDD}

Demographic and baseline pre-operative data are described in Table 1 . After $8.2 \pm 2.6$ years, there was no change in the UPDRS-II-OFF score $(p=0.66)$, S\&E-OFF score $(p=0.71)$ and the PDQ-39 summary index (SI) scores $(p=0.44)$, and sustained improvement in the UPDRS-IV score $(p \leq 0.0001)$. LEDD was considerably lower $(p<0.0001)$. Conversely, worsening was seen in the UPDRS-1 score $(p=0.0002)$, S\&E-ON score $(p<0.0001)$, and UPDRS-II-ON score $(p<0.0001)$ (Table 2$)$.

Table 1. Baseline Demographics and Pre-operative Data $(n=52)$.

\begin{tabular}{|c|c|}
\hline Baseline Characteristic & Mean (SD) Score \\
\hline Current age & $66.1(7.9)$ \\
\hline Years since DBS surgery & $8.2(2.6)$ \\
\hline PD duration at current time (years) & $19.7(5.6)$ \\
\hline PD duration at time of surgery (years) & $11.57(4.7)$ \\
\hline LEDD before surgery & $1326(652.5)$ \\
\hline Pre-operative UPDRS-I & $2.25(1.78)$ \\
\hline Pre-operative UPDRS-II, OFF & $20.82(5.47)$ \\
\hline Pre-operative UPDRS-II, ON & $8.27(5.24)$ \\
\hline Pre-operative UPDRS-III total, OFF, ON & $43.38(13.9), 16.86(9.36)$ \\
\hline Pre-operative UPDRS-III axial, OFF, ON & $11.52(4.51), 4.57(2.73)$ \\
\hline Pre-operative UPDRS-III rest tremor, OFF, ON * & $3(7), 0(0)$ \\
\hline Pre-operative UPDRS-III postural tremor, OFF, ON * & $1.5(2.5), 0(1)$ \\
\hline Pre-operative UPDRS-III bradykinesia left OFF, ON & $7.71(2.94), 3.27(2.27)$ \\
\hline Pre-operative UPDRS-III bradykinesia right OFF, ON & $6.69(2.46), 2.52(1.7)$ \\
\hline Pre-operative UPDRS-III rigidity OFF, ON & $9.4(4.33), 3.99(3.65)$ \\
\hline Pre-operative UPDRS-IV & $8.36(3.25)$ \\
\hline Pre-operative S\&E-OFF & $58 \%(20 \%)$ \\
\hline Pre-operative S\&E-ON & $85 \%(10 \%)$ \\
\hline Pre-operative PDQ-39 Summary Index & $33.01(12.1)$ \\
\hline Pre-operative PDQ-39, Mobility domain & $46.25(20.4)$ \\
\hline Pre-operative PDQ-39, ADLs domain & $44.53(16.16)$ \\
\hline Pre-operative PDQ-39, Emotional well-being domain & $28.65(18.51)$ \\
\hline Pre-operative PDQ-39, Stigma domain & $32.68(22.8)$ \\
\hline Pre-operative PDQ-39, Social support domain & $14.92(17.92)$ \\
\hline Pre-operative PDQ-39, Cognitive impairment domain & $23.52(15.1)$ \\
\hline Pre-operative PDQ-39, Communication domain & $26.39(19.93)$ \\
\hline Pre-operative PDQ-39, Bodily discomfort domain & $47.57(25.46)$ \\
\hline
\end{tabular}


Table 2. Change in Scores from Pre-DBS to Current Time ( $8.2 \pm 2.6$ years post-DBS).

\begin{tabular}{ccc}
\hline Outcome Measure & All Patients $(\boldsymbol{n}=\mathbf{5 2})$ & $p$-Value \\
\hline LEDD & $-634.8(721.4)$ & $<0.0001$ \\
UPDRS-I * & $1(2)$ & 0.0002 \\
UPDRS-II, OFF & $-0.59(9.3)$ & 0.66 \\
UPDRS-II, ON & $7.9(8)$ & $<0.0001$ \\
UPDRS-IV & $-4.6(3.4)$ & $<0.0001$ \\
S\&E-OFF & $-2.0 \%(32.0 \%)$ & 0.71 \\
S\&E-ON & $-17.0 \%(23.0 \%)$ & $<0.0001$ \\
PDQ-39, Summary Index & $2.1(18.2)$ & 0.44 \\
PDQ-39, Mobility domain & $9.7(31)$ & 0.03 \\
PDQ-39, ADLs domain & $-6.2(27.4)$ & 0.13 \\
PDQ-39, Emotional well-being domain & $1(24.7)$ & 0.77 \\
PDQ-39, Stigma domain & $-10.1(22.2)$ & 0.003 \\
PDQ-39, Social support domain & $6.3(26.9)$ & 0.11 \\
PDQ-39, Cognitive impairment domain & $4.6(22.8)$ & 0.16 \\
PDQ-39, Communication domain & $22.4(26.2)$ & $<0.0001$ \\
PDQ-39, Bodily discomfort domain & $-13.2(30.1)$ & 0.004 \\
\hline
\end{tabular}

Values represent means and SD. Values with * represent medians and IQR. For the UPDRS I, II, IV, and PDQ-39 higher scores indicate worse severity. A positive number indicates a worsened score from baseline, whereas a negative number indicates an improvement in score from baseline. For S\&E lower scores indicate worse severity. A positive number indicates an improvement from baseline, whereas a negative number indicates a worsening from baseline.

\subsection{Rush Patient-Centered Outcomes Questionnaire (RUSH-DBS-Q)}

Satisfaction was rated high by the majority with $65 \%$ of patients scoring a 0,1 or 2 evidenced by a median score (range) of $1(0-8)$. Patients endorsed having made the correct decision by undergoing DBS, with a score of $0(0-10)$, would choose to have DBS again, with a score of $0(0-10)$, and would recommend DBS to others, with a score of $0(0-10)$. The majority of patients indicated that they would have preferred surgery at an earlier time with $63.5 \%$ of patients scoring a 0,1 or 2, leading to a median score of 1 (0-10). (Figure 1).

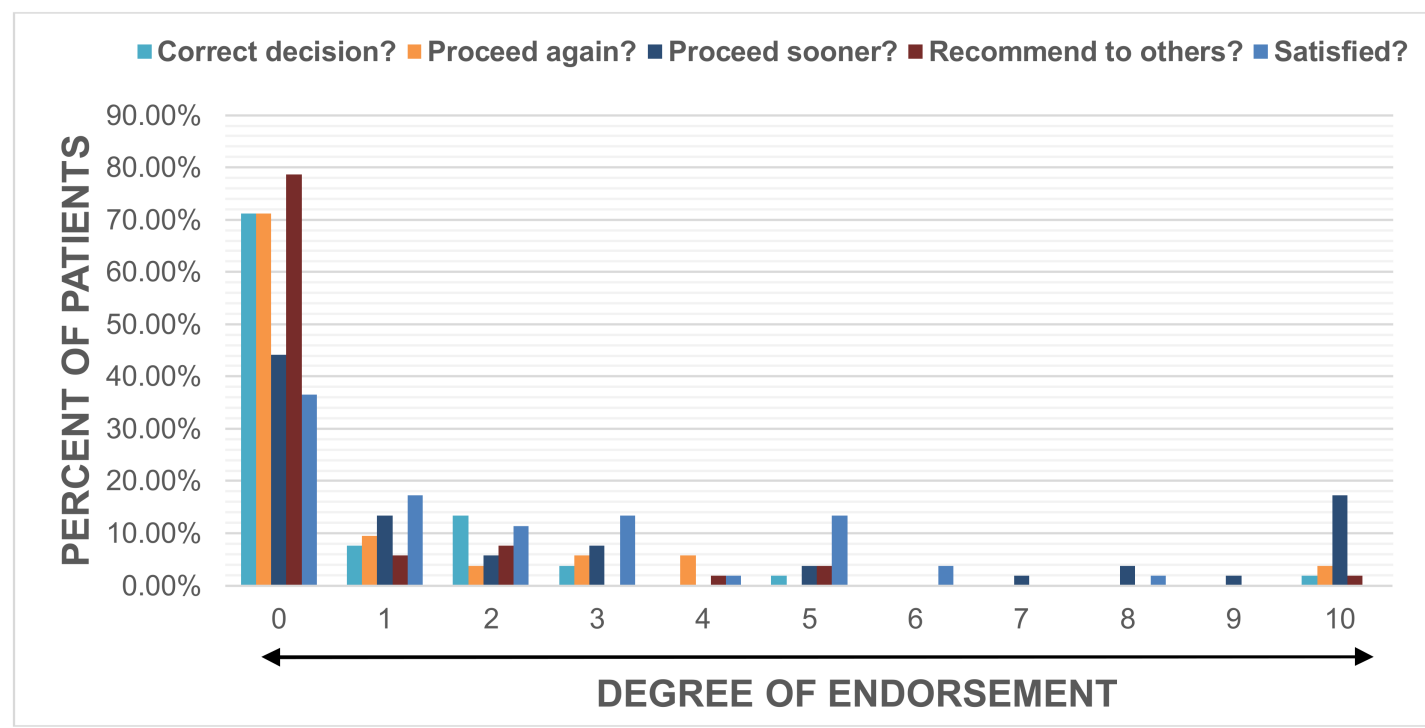

Figure 1. Patient endorsement. The percent of patients reporting degree of endorsement on the RUSHDBS-Q. All questions were scored from 0 to 10, whereby 0 represented the most 'positive' answer. The questions centered around making the correct decision to undergo DBS, theoretical decision to proceed again with DBS, timing of DBS, confidence in recommending DBS to others, and overall satisfaction with DBS. 
Worsening in patient-reported outcomes from pre-DBS to current time $(8.2 \pm 2.6$ years $)$ as measured by the PDQ-39 Summary Index $\left(r_{s}=0.57, p \leq 0.0001\right)$, PDQ-39 mobility domain $\left(r_{s}=0.57\right.$, $p \leq 0.0001)$, PDQ-39 emotional well-being domain $\left(r_{s}=0.40, p=0.005\right)$, PDQ-39 stigma domain $\left(r_{s}=0.40, p=0.005\right)$, PDQ-39 social support domain $\left(r_{s}=0.45, p=0.001\right)$, and S\&E-OFF $\left(r_{s}=-0.44\right.$, $p=0.002)$ correlated with lower satisfaction. There was no association between pre-operative levodopa responsiveness and satisfaction $\left(r_{S}=-0.01, p=0.93\right)$ (Table 3). There were no predictors of postoperative satisfaction found $(p=0.95)$ (Table S2).

Table 3. Correlation between Change in Pre-DBS Scales and Satisfaction.

\begin{tabular}{ccc}
\hline Outcome Measure & Spearman Correlation Coefficient & $p$-Value \\
\hline LEDD & 0.25 & 0.09 \\
UPDRS-I & 0.44 & 0.002 \\
UPDRS-II, OFF & 0.32 & 0.02 \\
UPDRS-II, ON & 0.21 & 0.14 \\
BPDeline (UPDR-III-OFF-UPDRS-III-ON) * & -0.01 & 0.93 \\
S\&E-OFF & 0.34 & 0.02 \\
S\&E-ON & -0.44 & 0.002 \\
PDQ-39, Summary Index & -0.34 & 0.02 \\
PDQ-39, Mobility domain & 0.57 & $<0.0001$ \\
PDQ-39, ADLs domain & 0.57 & $<0.0001$ \\
PDQ-39, Emotional well-being domain & 0.31 & 0.03 \\
PDQ-39, Stigma domain & 0.40 & 0.005 \\
PDQ-39, Social support domain & 0.40 & 0.005 \\
PDQ-39, Cognitive impairment domain & 0.45 & 0.001 \\
PDQ-39, Communication domain & 0.38 & 0.01 \\
PDQ-39, Bodily discomfort domain & 0.36 & 0.01 \\
\hline
\end{tabular}

Values represent correlation of current satisfaction with change in scales from pre-DBS to current time (8.2 \pm 2.6 years).

* Represents pre-operative levodopa response, UPDRS-III-OFF-UPDRS-III-ON.

\subsubsection{Expectations}

The pre-operative expectation target was set at a high level with $53 \%$ of patients scoring a 0,1 or 2 evidenced by a median score (range) of $2(0-10)$. The correlation between pre-surgical expectations and current satisfaction was not significant $\left(r_{s}=0.27, p=0.06\right)$.

\subsubsection{Patient-Rated Motor and Non-Motor Symptoms}

There was a weak but significant correlation between current satisfaction and patient-rated severity in motor $\left(r_{s}=0.36, p=0.01\right)$ and non-motor symptoms $\left(r_{s}=0.33, p=0.02\right)$ (Table S3). The majority of patients reported improvements in dyskinesias, motor fluctuations, tremor, rigidity and side effects of medication, whereas gait, balance and non-motor symptoms were mostly reported as being worse. (Figure 2, Table S4). Those who reported worsening in apathy $\left(r_{S}=0.4, p=0.03\right)$ and insomnia $\left(r_{s}=0.43, p=0.01\right)$ were less satisfied (Table S5). 


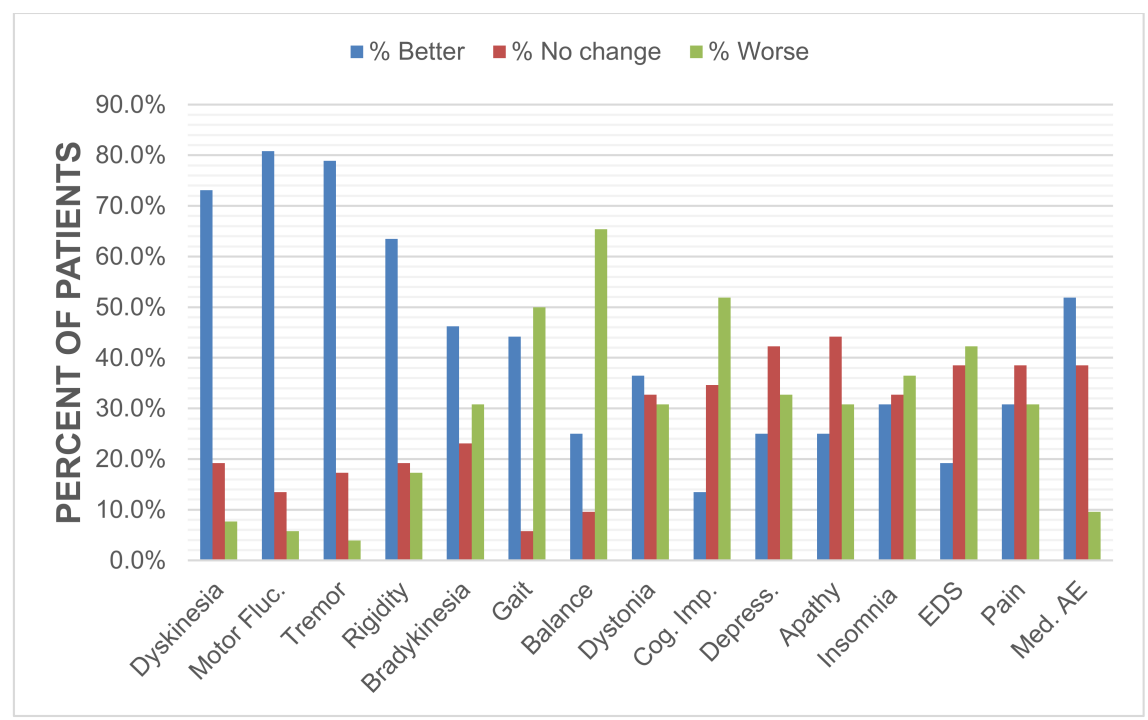

Figure 2. Change in self-rated symptom severity. All patients $(n=52)$ were asked to rate their PD motor and non-motor symptoms at two time points (pre-DBS and current time of $8.2 \pm 2.6$ years post-DBS). All items were rated based on severity, with 0 being the least severe and 10 being the most severe. Motor Fluc., motor fluctuations; Cog. Imp., cognitive impairment; EDS, excessive daytime sleepiness; Med AE, medication adverse effects.

\section{Discussion}

\subsection{Rush Patient-Centered Outcomes Questionnaire (RUSH-DBS-Q)}

To the best of our knowledge, this is the first study evaluating patients' and caregivers' satisfaction with DBS outcome in the long term (>5 years). Patient and caregiver perception is important given the elective nature of DBS surgery, especially in the context of a progressive neurological disorder. In this sample, the vast majority of patients did report satisfaction with surgery in the long term, despite their high pre-operative expectation target. While intuitively pre-operative expectations and satisfaction should be correlated, we did not find such relationship. Hasegawa et al. [6] did find a strong correlation between expectations and satisfaction in the short term but had a different methodological approach. In the future, we will adjust our questionnaire to better capture this relationship. The relationship between pre-operative expectations and post-operative satisfaction in this population remains unclear but the absence of a correlation does not negate the need to identify individualized expectations so that patients and caregivers can be adequately counseled regarding short- and long-term goals from DBS.

\subsection{Mobility}

Worsened mobility domain scores on the PDQ-39 correlated with lower satisfaction of DBS. Axial symptoms can be resistant to DBS in the long term and can have a significant impact on patient independence and QOL [9-11]. Maier et al. [12] found an inverse correlation between pre-operative axial scores and patient satisfaction with DBS at 3 months post-surgery. We did not find such association in our population possibly due to pre-operative screening methods at our institution.

\subsection{Non-Motor Symptoms}

Non-motor symptoms have a substantial impact on PD patients' QOL [13]. The effect of DBS on non-motor symptoms has been evaluated at 24 months [14] but is not established in the long term. A worsening can have a negative impact on perceived benefit [15]. As discussed by Joint and Aziz [16], patients and caregivers may focus on the motor outcomes after surgery and fail to consider the influence 
non-motor symptoms may have on QOL. In this sample, a significant worsening in certain non-motor symptoms correlated with less satisfaction. In particular, apathy was associated with decreased patient satisfaction. It has been suggested that apathy post-STN DBS surgery could be directly related to the dramatic reduction in dopaminergic medications [17]; however, in our patient population, there was no correlation between change in LEDD and apathy. A likely explanation is that patients with a larger reduction in medication have a superior benefit of DBS or, alternatively, that patients having a higher dopaminergic medication have progressed at a relatively faster rate. There were no pre-operative predictors of post-operative apathy identified and pre-operative neuropsychological testing did not reveal a significant level of depression in any given patient.

\subsection{Study Limitations}

There are limitations in our study worth noting. We used a locally developed, non-validated questionnaire to evaluate patient-centered outcomes. However, at the time the study was completed, no such instrument was available. In addition, the RUSH-DBS-Q was not completed pre-operatively, and all answers were obtained at the time of survey, more than eight years after surgery. We partially addressed partial recall by asking patients and caregivers to agree on answers to limit each individual's recall bias. In addition, we did not repeat the UPDRS-III at the time of survey. This would have lent to a better understanding of the relationship between patient perception and motor outcome. Finally, all patients included in the study were under the care of one treating neurologist. Given the long follow-up since surgery, patients were likely satisfied with their care which could have led to bias when answering the satisfaction questions. However, to limit this bias, the treating neurologist was not involved in administration of the questionnaires.

\section{Conclusions}

To our knowledge, this is the first study to assess long-term patient and caregiver satisfaction with STN DBS. After an average of eight years post-DBS surgery, the majority of patients were satisfied, felt they had made the correct decision to undergo DBS, would choose to have DBS again, would recommend DBS to others, and would have preferred to have surgery sooner. Pre-operative expectations were higher than expected based on our conservative pre-operative counseling, but they did not correlate with satisfaction. The latter should not negate the necessity of setting realistic expectations to caregivers and patients. We are currently collecting data prospectively to confirm the results of this pilot study.

Supplementary Materials: The following are available online at http:/ / www.mdpi.com/2076-3425/8/4/60/s1, Table S1:Correlation of Change in Reported Symptom and Satisfaction, Table S2: Patient Reported Symptom Severity, Table S3: Locally developed DBS patient centered outcome questionnaire (RUSH-DBS-Q), Table S4: Correlation of Baseline Motor Scores and Satisfaction, Table S5:Correlation of Reported Symptom Severity and Satisfaction.

Acknowledgments: The Rush Movement Disorder Center is a designated Parkinson's Disease Foundation Parkinson's Research Center and a National Parkinson Foundation Center of Excellence.

Author Contributions: Jessica A. Karl research project: conception, organization and execution; statistical analysis: design, execution, review, critique; manuscript: writing of the first draft, review, critique. Bichun Ouyang statistical analysis: design, execution, review, critique. Kalea Colletta research project: conception, organization and execution. Leo Verhagen Metman research project: conception; statistical analysis: review, critique; manuscript: review, critique.

Conflicts of Interest: No authors have any conflicts of interest pertaining to this manuscript.

\section{References}

1. Castrioto, A.; Lozano, A.M.; Poon, Y.Y.; Lang, A.E.; Fallis, M.; Moro, E. Ten-year outcome of subthalamic stimulation in Parkinson disease: A blinded evaluation. Arch. Neurol. 2011, 68, 1550-1556. [CrossRef] [PubMed]

2. Zibetti, M.; Merola, A.; Rizzi, L.; Angrisano, S.; Azzaro, C.; Artusi, C.A.; Arduino, N.; Marchisio, A.; Lanotte, M.; Rizzone, M. Beyond nine years of continuous subthalamic nucleus deep brain stimulation in Parkinson's disease. Mov. Disord. 2011, 26, 2327-2334. [CrossRef] [PubMed] 
3. Rizzone, M.G.; Fasano, A.; Daniele, A.; Zibetti, M.; Merola, A.; Rizzi, L.; Piano, C.A.; Piccininni, C.; Romito, L.M.; Lopiano, L.; et al. Long-term outcome of subthalamic nucleus DBS in Parkinson's disease: From the advanced phase towards the late stage of the disease? Parkinsonism Relat. Disord. 2014, 20, 376-381. [CrossRef] [PubMed]

4. Siderowf, A.; Jaggi, J.L.; Xie, S.X.; Zibetti, M.; Merola, A.; Rizzi, L.; Piano, C.A.; Piccininni, C.; Romito, L.M.; Lopiano, L.; Albanese, A.L. Long-term effects of bilateral subthalamic nucleus stimulation on health-related quality of life in advanced Parkinson's disease. Mov. Disord. 2006, 21, 746-753. [CrossRef] [PubMed]

5. Volkmann, J.; Albanese, A.; Kulisevsky, J.; Tornqvist, A.L.; Houeto, J.L.; Pidoux, B.; Bonnet, A.M.; Mendes, A.; Benabid, A.L.; Fraix, V.; et al. Long-term effects of pallidal or subthalamic deep brain stimulation on quality of life in Parkinson's disease. Mov. Disord. 2009, 24, 1154-1161. [CrossRef] [PubMed]

6. Hasegawa, H.; Samuel, M.; Douiri, A.; Ashkan, K. Patients' expectations in subthalamic nucleus deep brain stimulation surgery for Parkinson disease. World Neurosurg. 2014, 82, 1295-1299. [CrossRef] [PubMed]

7. Frank, L.; Basch, E.; Selby, J.V. Patient-Centered Outcomes Research Institute. The PCORI perspective on patient-centered outcomes research. JAMA 2014, 312, 1513-1514. [CrossRef] [PubMed]

8. Stebbins, G.T.; Goetz, C.G. Factor structure of the unified Parkinson's disease rating scale: Motor examination section. Mov. Disord. 1998, 13, 633-636. [CrossRef] [PubMed]

9. Fasano, A.; Romito, L.M.; Daniele, A.; Piano, C.; Zinno, M.; Bentivoglio, A.R.; Albanese, A. Motor and cognitive outcome in patients with Parkinson's disease 8 years after subthalamic implants. Brain 2010, 133, 2664-2676. [CrossRef] [PubMed]

10. Gervais-Bernard, H.; Xie-Brustolin, J.; Mertens, P.; Polo, G.; Klinger, H.; Adamec, D.; Broussolle, E.; Thobois, S. Bilateral subthalamic nucleus stimulation in advanced Parkinson's disease: Five year follow-up. J. Neurol. 2009, 256, 225-233. [CrossRef] [PubMed]

11. Krack, P.; Batir, A.; Van Blercom, N.; Chabardes, S.; Fraix, V.; Ardouin, C.; Koudsie, A.; Limousin, P.D.; Benazzouz, A.; LeBas, J.F.; Benabid, A.L. Five-year follow-up of bilateral stimulation of the subthalamic nucleus in advanced Parkinson's disease. New Engl. J. Med. 2003, 349, 1925-1934. [CrossRef] [PubMed]

12. Maier, F.; Lewis, C.J.; Horstkoetter, N.; Eggers, C.; Kalbe, E.; Maarouf, M.; Kuhn, J.; Zurowski, M.; Moro, E.; Woopen, C.; et al. Patients' expectations of deep brain stimulation, and subjective perceived outcome related to clinical measures in Parkinson's disease: A mixed-method approach. J. Neurol. Neurosurg. Psychiatry. 2013, 84, 1273-1281. [CrossRef] [PubMed]

13. Martinez-Martin, P. The importance of non-motor disturbances to quality of life in Parkinson's disease. J. Neurol. Sci. 2011, 310, 12-16. [CrossRef] [PubMed]

14. Dafsari, H.S.; Silverdale, M.; Strack, M.; Rizos, A.; Ashkan, K.; Mahlstedt, P.; Sachse, L.; Steffen, J.; Dembek, T.A.; Visser-Vandewalle, V.; et al. Nonmotor symptoms evolution during 24 months of bilateral subthalamic stimulation in Parkinson's disease. Mov. Disord. 2018, 33, 421-430. [CrossRef] [PubMed]

15. Klingelhoefer, L.; Samuel, M.; Chaudhuri, K.R.; Ashkan, K. An update of the impact of deep brain stimulation on non-motor symptoms in Parkinson's disease. J. Parkinsons Dis. 2014, 4, 289-300. [PubMed]

16. Joint, C.; Aziz, T.Z. Outcome after deep brain stimulation surgery of the subthalamic nucleus for Parkinson disease: Do we understand what is important to our patients? World Neurosurg. 2014, 82, 1035-1036. [CrossRef] [PubMed]

17. Tremblay, L.; Worbe, Y.; Thobois, S.; Sgambato-Faure, V.; Feger, J. Selective dysfunction of basal ganglia sub-territories: From movement to behavioral disorders. Mov. Disord. 2015, 30, 1155-1170. [CrossRef] [PubMed]

(C) 2018 by the authors. Licensee MDPI, Basel, Switzerland. This article is an open access article distributed under the terms and conditions of the Creative Commons Attribution (CC BY) license (http:/ / creativecommons.org/licenses/by/4.0/). 